\begin{tabular}{l|l} 
REVISTA & $\begin{array}{l}\text { Revista Educación } \\
\text { ISSN: 0379-7082 } \\
\text { ISSN: 2215-2644 } \\
\text { revedu@ gmail.com } \\
\text { Universidad de Costa Rica } \\
\text { Costa Rica }\end{array}$
\end{tabular}

\title{
Aplicación del Aprendizaje Basado en Problemas (ABP) de John Barell en la comprensión literal
}

Hurtado Serna, Mirian; Salvatierra Melgar, Ángel

Aplicación del Aprendizaje Basado en Problemas (ABP) de John Barell en la comprensión literal

Revista Educación, vol. 44, núm. 2, 2020

Universidad de Costa Rica, Costa Rica

Disponible en: http://www.redalyc.org/articulo.oa?id=44062184013

DOI: https://doi.org/10.15517/revedu.v44i2.38256

Esta obra está bajo una Licencia Creative Commons Atribución-NoComercial-SinDerivar 3.0 Internacional. 


\title{
Aplicación del Aprendizaje Basado en Problemas (ABP) de John Barell en la comprensión literal
}

\author{
Application of Problem Based Learning (ABP) by John Barell in literal understanding
}

Mirian Hurtado Serna

Universidad Cesar Vallejo, Perú

hurtadoser22@gmail.com

iD http://orcid.org/0000-0003-2895-081X

Angel Salvatierra Melgar

Universidad Nacional Mayor de San Marcos, Perú

asalvatierram@unmsm.edu.pe

(iD http://orcid.org/0000-0003-2817-630X
DOI: https://doi.org/10.15517/revedu.v44i2.38256

Redalyc: http://www.redalyc.org/articulo.oa?id=44062184013

Recepción: 29 Julio 2019

Aprobación: 14 Abril 2020

\section{Resumen:}

El estudio puesto a consideración, explica la importancia del aprendizaje basado en problemas, este fue desarrollado con base en el enfoque cuantitativo, de diseño cuasi experimental, el objetivo fue verificar los efectos de la aplicación del aprendizaje basado en problemas (ABP) de John Barell en la comprensión literal en los escolares del nivel primario del $4^{\circ}$ grado de la Institución Educativa del Perú. Para conocer los efectos del ABP se le planteó al estudiantado del grupo experimental: retos, curiosidades, incertidumbre sobre los fenómenos o problemas de la vida cotidiana a través de las tres unidades del ABP, siguiendo los diez pasos John Barell de manera secuencial con la finalidad de motivar al alumnado para que se involucre en la lectura al buscar información que le permita resolver los retos planteados, mientras que el grupo de control desarrolló sus clases de manera habitual. Los resultados obtenidos, muestran que el $74,07 \%$ de estudiantes del grupo experimental se encuentran en el nivel de logro destacado, y el 25,93\% en el logro esperado frente al 14,81\% del grupo control que se encuentra en nivel de logro esperado y el 85,2\% en los dos niveles más bajos (proceso e inicio), esta comparación se evidenció por la prueba de U de Mann-Whitney. cuyo resultado de contraste fue $\mathrm{p}<0.05$. Ello permitió afirmar que el aprendizaje basado en Problemas de Jonh Barrel tiene efecto significativo en la comprensión literal.

Palabras Clave: Aprendizaje basado en problemas, Comprensión literal, Situación retadora, John Barell.

\section{Abstract:}

The study for consideration shows the importance of problem-based learning that was developed under the quantitative approach, of quasi-experimental design, the objective was to verify the effects of the application of literal problem-based learning (ABP) in schoolchildren of the level Primary of the 4th grade of the Educational Institution of Peru. In order to know the effects of the ABP, the students of the experimental group were asked: challenges, curiosities, uncertainty about the phenomena or problems of daily life through the three units of the ABP, following the ten steps John Barell sequentially with the In order to motivate students to become involved in reading by looking for information that allows them to solve the challenges posed, while the control group developed their classes on a regular basis. The results obtained show that $74,07 \%$ of the students in the experimental group are at the outstanding achievement level, and $25,93 \%$ in the expected achievement compared to $14,81 \%$ of the control group who are at the expected achievement level and $85,2 \%$ in the two lower levels (process and onset), this comparison was evidenced by the Mann-Whitneya $U$ test whose contrast results were $\mathrm{p}<0.05$. Who allowed to affirm that the problem-based learning of John Barrel has a significant effect on literal understanding.

KEYWORDS: Problem-based learning, Literal comprehension, Challenging situation, John Barell.

\section{INTRODUCCIÓN:}

Entre los desafíos de la educación se encuentra el mejorar la comprensión de textos en estudiantes que están ubicados en los distintos niveles, lo cual es la capacidad que se posee para interpretar lo que se lee, 
así como en referencia al significado de las palabras y frases que forman un texto o en relación con la comprensión integral en todo un escrito, como lo incrementa Vidal y Manriquez (2016), la lectura es como un ingrediente primordial para la comprensión global y perfeccionar su pericia en la lectura comprensiva. Fonseca, Migliardo, Simian, Olmos, y León (2019), avalan que es una condición para el aprendizaje y el desenvolvimiento en cualquier función que se desarrolla, bajo este aporte se puede decir que cada estudiante que comprende una lectura es más competente, al tener mayor solvencia en el manejo de la información por la tendencia del trabajo que se tiene con gran cantidad de información. Peña (2019) menciona la importancia del gozo por la lectura para amparar la comprensión, teniendo presente que el proceso lector implica habilidades, vivencias y experiencias. Ante lo citado se complementa que el lector elabora un significado con los pensamientos importantes del texto y los va relacionando con los conceptos ya poseídos, esto le permite interactuar con el texto que lee y muchas veces no toma en cuenta la amplitud del párrafo o el texto completo. Pinzas (2017) ratifica que es un proceso (a) interactivo, porque el conocimiento ofrecido por el texto dialoga con la información previa que tiene el lector; (b) constructivo, porque se presenta en la combinación de lo que evoca el lector y lo que ofrece el material impreso, es decir, no se da en el vacío este proceso, es incesante y cada vez más complejo según se transite por los niveles de la comprensión.

En la comprensión literal, el primer propósito del lector es sacar la información que el autor ha referido de manera directa o explicita, es decir que la comunicación se encuentra plasmado entre las líneas del texto. Pinzas (2003), manifiesta que en este nivel, se localiza la información que se exhibe ubicada en cualquier parte del texto y a la vez, es el nivel básico que soporta los niveles superiores de la comprensión. Como lo ratifica Durango (2017), la persona lectora no profundiza se apoya en la información otorgada de primera mano, sin escudriñar en factibles interpretaciones más allá de su sentido. Rojas y Cruzata (2016) expresan que la intención es explorar los eventos tal como aparecen en el texto, identificando los protagonistas, el espacio de los hechos y el tiempo en el cual acontecen las acciones.

Conociendo las exigencias de nivel literal bajo los distintos aportes se puede decir que estudiantes del $4^{\circ}$ grado de primaria no distinguen lo que lee como lo demuestran las evaluaciones censales, donde muchos permanecen en los dos niveles más bajos de logros de aprendizaje, como lo menciona el Ministerio de Educación del Perú (2018), donde el alumnado del $4^{\circ}$ en el año 2016 el 9,1\% se ubica en nivel previo al inicio y el 26,2\% en inicio y en el año 2018 el 10,15\% en previo al inicio y el 24,2\% en inicio según la evaluación censal en comprensión lectora a nivel nacional. Como lo sustenta Zeballos y Nyquist (2017), leer, es mucho más que traducir un código, comprendiendo que no todos los textos presentan los mismos obstáculos en la interacción entre el lector. De Mier, Amado, y Benítez (2015) revelan que las lecturas más habituales en los hogares y los centros educativos son los textos narrativos y al mismo tiempo manifiesta que en estas últimas décadas, se ha emprendido a tomar en consideración la comprensión de textos expositivos que impulsan un desempeño alto en la recuperación y comprensión del testimonio leído; porque abordan conocimientos científicos.

El presente estudio al estudiantado se le presenta todo tipo de textos, se tiene en cuenta su propósito lector, para resolver las situaciones problemáticas que se plantea en el Aprendizaje Basado en Problemas (ABP) de John Barell (2007), que es un método de exploración muy beneficioso para resolver preguntas, curiosidades o las indecisiones acerca de los fenómenos complicados de la vida. Barell (2007), declara que es una incógnita que exige una solución y esto admite desafiar al estudiante en la exploración del conocimiento para responder sus propias interrogantes y no solo al desafío que proyecta el/la docente, por estar centrado en un enfoque investigativo, al igual que Marra, Jonassen, Palmer y Luft (2014), manifiestan que ABP es "un tipo de metodología activa, de enseñanza, centrada en el/la estudiante, que se caracteriza por producir el aprendizaje del/la estudiante en el contexto de la solución de un problema auténtico" (p. 221).

En la aplicación del trabajo el/la docente asume el rol de guiar a cada estudiante en su enseñanza, para alcanzar los retos planteados, promoviendo sus habilidades y autonomía con menos dependencia del formador, fomentando que entre sus pares discutan los caminos por seguir para resolver los retos tal cual lo manifiesta. Morales (2018), indica que los miembros interactúan entre sí, compartiendo sus puntos de vista, 
aprendiendo a trabajar colaborativamente y llegando a deducciones válidas. Rodríguez y Fernández (2017), sustentan que, en este desafío, el alumnado podrá habilitarse de los materiales que consideren pertinentes para alcanzar su objetivo. Sastoque, Ávila, y Olivares (2016), declaran que al trabajar de esta forma se promociona el descubrimiento y perfeccionamiento de sus habilidades, como el pensamiento crítico y la evaluación de manera reflexiva en situaciones reales de su contexto.

Es necesario proponer al estudiante actividades retadoras como lo menciona la metodología de ABP, teniendo en cuenta la secuencia de los diez pasos en la planificación de las unidades como se propone a continuación. Barell (2007): 1) En este paso el/la docente selecciona el contenido según el currículo, sin dejar de lado el intereses del/la estudiantes, es decir se les plantea situaciones auténticas de aprendizaje. Rigo y Donolo (2017), incrementa que el valor del beneficio del conocimiento escolarizado es contextualizar a la vida cotidiana; 2) Se tiene graficar todos los elementos probables del tema, haciendo uso de organizadores gráficos, para darle mayor visualidad de la temática que se abordará, con la finalidad de facilitar el aprendizaje del/la estudiante, las bondades expuestas se ratifican con otro sustentos. Toro y Buitrago (2018), exponen que los organizadores gráficos son herramientas que posibilitan la representación de conocimientos y apoyan en los procesos cognitivos. Novoa, Cancino, Flores y Nieto (2018), sustentan que los mapas mentales armónicos son potentes ejecutantes psicológicos para leer, su efecto estimula el hemisferio derecho e izquierdo del cerebro y, Nunura (2018), manifiesta que son estrategias eficientes, que admiten mejorar la comprensión literal, inferencial y crítico, 3) En este paso el/la docente decide los elementos o sub temas a incluir en la planificación de unidades del ABP, teniendo en cuenta las áreas relacionadas, los intereses y las vivencias del/la estudiante que puedan ser significativos para ellos. Como lo incrementa Carranza y Caldera (2018), un contenido motivador nace de un compromiso auténtico en el proceso de aprendizaje del escolar; 4) Se trazan los objetivos que deben ser relevantes, desafiantes y que promuevan la alta demanda cognitiva en las y los estudiantes. Para Ramos y Casas (2018), es vital valorar la demanda cognitiva de una actividad, para entender el sacrificio mental que se supone utilizar para poder resolver la actividad. Por ello se menciona que la dificultad del conocimiento o demanda cognitiva es un modo de poder clasificar el aprendizaje que se quiere conseguir según el objetivo trazado, teniendo cuenta que son retadoras pero a la vez factible de lograr para evitar caer en la desmotivación del/la estudiante; 5) En este paso se determina una pregunta importante o una situación problemática que valga la pena que las y los estudiantes piensen durante un tiempo significativo para desarrollar preguntas y reflexiones sostenidas. López, Veit y Solano (2014), indican que es importante tener presente en la calidad de preguntas que efectúa cada docente para la reflexión y además complementa que el aprendiz debe dominar la formulación de las preguntas, porque es una estrategia cognitiva que promueve las habilidades de orden superior; 6) En este paso se diseña estrategias a largo plazo (experiencias de aprendizaje) que permitan alcanzar los objetivos, estimulando la resolución de problemas difíciles, mediante el desarrollo del pensamiento crítico o creativo. Para este fin cada docente debe gestionar el acompañamiento pertinente, que motive a la obtención del buen producto. Malla (2016) declara que la tarea del profesorado es guiar al estudiantado para un aprendizaje consciente y autónomo; 7) En este paso el personal docente debe seguir creando experiencias de aprendizaje, utilizando un marco de experiencias específicas iniciales, centrales y culminantes; 8) Al llegar a este paso, se debe disponer de experiencias que faculten la evaluación del aprendizaje, teniendo en cuenta la planificación inicial, los objetivos y los conocimientos generados. Sánchez (2018) asevera que la evaluación persistente y técnicamente conveniente ayuda a entender el efecto de la enseñanza y el aprendizaje 9) En el penúltimo paso cada docente debe ofrecer oportunidades para la transferencia y la aplicación de lo aprendido en la unidad, poniendo en práctica lo aprendido bajo otras circunstancias. Como lo refiere Souza, Pfeiffer y Mendes de Oliveira (2015), la lección aprendida, debe ser generalizada a otros contextos o condiciones opuestas al de la posición inicial; 10) En este último paso, docentes y estudiantes ejecutan la reflexión metacognitiva sobre el proceso desarrollado, con base en preguntas y respuestas, con la finalidad de reflexionar sobre sus pensamientos y sentimientos. 
Flavell (1979); Tamayo, Cadavid, y Montoya (2019), exponen que el conocimiento metacognitivo se aclara primordialmente al conocimiento que posee el sujeto acerca de sus progresos cognitivos.

Por todo lo descrito, se lleva a cabo la investigación cuasi experimental la cual responde al problema de investigación: ¿Cuál es el efecto del ABP de John Barell en la comprensión literal en los escolares del nivel primario del $4^{\circ}$ grado de la Institución Educativa del Perú? Para ello se le planteó al estudiantado del grupo experimental actividades significativas que generen retos, curiosidades, dudas incertidumbre sobre los fenómenos o problemas de la vida cotidiana, siguiendo los diez pasos del ABP John Barell que trabaja bajo un enfoque investigativo, con la finalidad de motivar al estudiante a que investigue a través de la lectura de diversas fuentes que le ayuden a resolver los retos planteados. Mientras que el grupo de control desarrolla sus clases con la misma metodología habitual y al finalizar las tres unidades planteadas en la investigación se podrá verificar los efectos de $\mathrm{ABP}$ en la comprensión literal al contrastar la hipótesis del estudio.

\section{MÉTODOS Y MATERIALES}

El estudio se fundamenta dentro del enfoque cuantitativo de diseño experimental de tipo cuasi experimental Campbell y Stanley (1995), distribuidos en dos grupos; grupo de control (27) y el otro experimental (27) del $4^{\circ}$ de primaria correspondiente al IV ciclo de la Educación Básica Regular, seleccionado de manera intencional no probabilística de una población de 520 estudiantes, el estudio se desarrolló con la ejecución de 21 sesiones. El instrumento que mide la comprensión literal tiene 21 ítems distribuidos de la siguiente manera: 6 de respuesta abierta, 4 de respuesta cerrada, 2 verdadero - falso y 9 de alternativa múltiple las cuales fueron aplicados en el pre test y post test de los grupos de estudio. El análisis de logro, se dio según los rangos de puntuación de Inicio, Proceso, Logro esperado y Logro destacado de acuerdo al sistema de calificación peruana. El instrumento aplicado se sustentó en las bases teóricas de Pinzas (2003), quien menciona las pautas que se tienen que seguir para redactar las preguntas del nivel literal como: Responder a las preguntas Qué, quién, dónde, cuándo, con quién, cómo, y entre otras interrogantes, para formular preguntas directas con la finalidad de recoger la información, se planteó oraciones afirmativas para ver si van con el texto y se consideraron oraciones incompletas. El instrumento elaborado fue sometido a una muestra piloto obteniendo los siguientes resultados: a) La validez por criterio de jueces con la técnica de V de Aiken alcanzó el $0.84^{* *}$. b) para la confiablidad interna se procedió con la técnica de Kuder y Richarson $\mathrm{R}_{20}$ por la naturaleza de índice dicotómico alcanzando 0.8340 demostrando moderado nivel de confiabilidad y el nivel de complejidad de 66,87\% según la correspondencia a tabla del índice adecuada; Campos, Contreras, Riffo, y Véliz (2014), asimismo se tiene la validez predictiva Ruvalcaba, Gallegos, Orozco, y Bravo (2019) la cual tiene la capacidad de pronosticar los acontecimientos futuros, alcanzó 0,8965, significando que cada estudiante tiene la misma certeza de responder similar a la muestra piloto. Para el análisis de datos se presentan las tablas de frecuencia y la demostración de U de Mann-Whitney; para la confrontación de los dos grupos, y el examen de Kruskal-Wallis para la comparación múltiple. Estas pruebas se efectuaron a razón de que los datos no presentaron la distribución normal.

\section{Resultados}

Se muestra los efectos de la aplicación del Aprendizaje Basado en Problemas de John Barell en la comprensión literal a través de la comparación del pre test y post test en ambos grupos de estudio, como se observa en la Figura 1. 


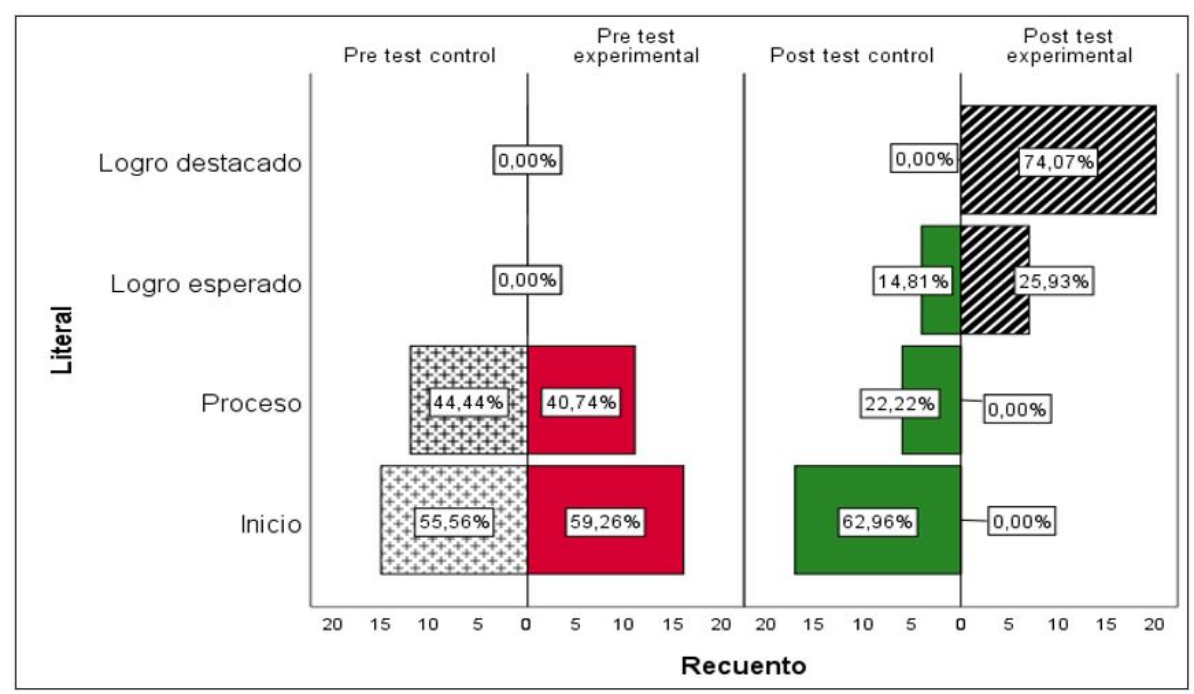

FIGURA 1

Conclusiones de la comprensión literal en los escolares del $4^{\circ}$ de la Institución Educativa de Vitarte, 2019 Fuente: Elaboración propia

La imagen permitió visualizar los hallazgos de la investigación, en el pre test, más del $50 \%$ de las y los estudiantes se ubican en el nivel de inicio, como el 55,56\% del grupo de control y el 59,29\% grupo del experimental. Estos resultados demuestran que los aprendices no son capaces de identificar detalles explícitos que están en el texto como: completar oraciones o responder interrogantes: Por ejemplo, Celestino es un... (Músico), ¿Quiénes disfrutan de la música? (el público); tampoco discriminan la secuencia del texto y, mucho menos, reconocen el contenido principal o secundario, sin embargo, el 44,44\% del grupo control y en el 40,74\% del experimental se ubican en el nivel de proceso, ellos pueden identificar detalles o discriminar la secuencia del texto, pero demuestran dificultad para reconocer el contenido principal o secundario. Es pertinente mencionar que nadie del estudiantado alcanzó los dos niveles superiores, como el logro esperado y logro destacado.

Después de la aplicación del Aprendizaje Basado en Problemas de John Barell (Resultados del post test) se observó que el 74,07\% del grupo experimental se ubican en nivel más alto, como el logro destacado, demostrando el empoderamiento de los indicadores correspondientes al nivel literal considerados en el estudio, en comparación al grupo de control que no alcanza las exigencias de este nivel.

En el siguiente nivel de logro esperado se ubica el 25,93\% de estudiantes del grupo experimental y solo el 14,81\% del grupo de control y el 85,18\% aún permanecen en las dos escalas de logros más bajo. Frente a los resultados obtenidos la aplicación del Aprendizaje Basado en Problemas fue significativo para la comprensión literal, porque que la metodología empleada bajo el enfoque investigativo permitió que estudiantes lean textos ubicando la información explícita que les ayude a aclarar sus dudas e incertidumbres al desarrollar el análisis de la información en forma individual o colectiva.

\section{Resultado de la hipótesis}

Para dar a conocer el Nivel de significación del ABP en la comprensión literal, se aplicó al estadístico de U de Mann-Whitney. para la muestras independientes y la comparación entre el pre test y post test. Al efecto, la hipótesis nula del estudio muestra que el aprendizaje Basado en Problemas de Jonh Barrel no tiene efecto en la comprensión literal. Para su contraste, se tienen los resultados en la Tabla 1 y su descripción. 
TABLA 1

Análisis de acuerdo al nivel de significación la hipótesis

\begin{tabular}{|c|c|c|c|c|c|c|c|c|c|}
\hline \multicolumn{10}{|c|}{ tes } \\
\hline & & & $\begin{array}{l}\text { Pre test } \\
\text { control }\end{array}$ & $\begin{array}{l}\text { Pre test } \\
\text { experimental }\end{array}$ & Parámetros & $\begin{array}{l}\text { Post } \\
\text { test } \\
\text { control }\end{array}$ & $\begin{array}{l}\text { Post test } \\
\text { experimental }\end{array}$ & TOTAL & Parámetros \\
\hline \multirow{9}{*}{ Literal } & & Recuento & 15 & 16 & & 17 & 0 & 48 & \multirow{10}{*}{$\begin{array}{l}U= \\
0.00 \\
z= \\
6.366 \\
\text { p_valor } 0.00\end{array}$} \\
\hline & Inlc10 & $\%$ dentro & $55,6 \%$ & $59,3 \%$ & & $63,0 \%$ & $0,0 \%$ & $44,4 \%$ & \\
\hline & & Recuento & & 11 & & 6 & & & \\
\hline & Fivesso & $\%$ dentro & $44,4 \%$ & $40,7 \%$ & $\mathrm{U}=$ & $22,2 \%$ & $0,0 \%$ & $26,9 \%$ & \\
\hline & Logro & Recuento & 0 & 0 & 364.00 & 4 & & & \\
\hline & esperado & $\%$ dentro & $0,0 \%$ & $0,0 \%$ & $z=$ & $14,8 \%$ & $25,9 \%$ & $10,2 \%$ & \\
\hline & Logro & Recuento & 0 & 0 & 0.009 & 0 & & & \\
\hline & destacado & $\%$ dentro & $0,0 \%$ & $0,0 \%$ & P_valor 0.993 & $0,0 \%$ & $74,1 \%$ & $18,5 \%$ & \\
\hline & & Recuento & 27 & 27 & & 27 & 27 & 108 & \\
\hline Total & & $\begin{array}{l}\text { \% dentro } \\
\text { de Test }\end{array}$ & $100,0 \%$ & $100,0 \%$ & & $100,0 \%$ & $100,0 \%$ & $100,0 \%$ & \\
\hline
\end{tabular}

Fuente: Elaboración propia

Los resultados que se muestran en la tabla, relativamente los porcentajes obtenidos por cada nivel son similares en el pre test, sin embargo, la prueba de $U$ de Mann-Whitney. cuyo resultado fue $\mathrm{p}>0,05$ determina que no hubo ninguna significación de comparación antes de la experimentación. Después de la aplicación de las sesiones experimentadas los resultados muestran diferencia en comparaciones porcentuales entre ambos grupos, tal es el caso que ningún estudiante del grupo control y el 74,1\% del grupo experimental se encuentran en logro destacado, permitiendo asumir al resultado de la prueba $U$, donde zc < zt $(-6,366<-1,96)$ además el valor_p $=0,000$ es inferior al $\alpha=0,05$, permitiendo afirmar que el aprendizaje Basado en Problemas de Jonh Barrel tienen efecto en la comprensión literal, ver Tabla 2.

TABLA 2

Comparación porcentual entre grupos de las variables latentes por índices

\begin{tabular}{|c|c|c|c|c|}
\hline \multirow[b]{2}{*}{$\begin{array}{l}\text { Variables } \\
\text { latentes }\end{array}$} & \multirow[b]{2}{*}{ Índices } & \multirow[b]{2}{*}{ fi } & \multicolumn{2}{|l|}{ Grupo } \\
\hline & & & $\begin{array}{l}\text { Grupo } \\
\text { experimental }\end{array}$ & $\begin{array}{l}\text { Grupo } \\
\text { control }\end{array}$ \\
\hline \multirow{3}{*}{ Lee en casa } & \multirow{3}{*}{$\begin{array}{l}\text { No lee en casa } \\
\text { Algunas veces lee en } \\
\text { casa } \\
\text { Todos los dias lee en } \\
\text { casa }\end{array}$} & $\% \mathrm{fi}$ & $22,2 \%$ & $18,5 \%$ \\
\hline & & $\% \mathrm{fi}$ & $74,1 \%$ & $74,1 \%$ \\
\hline & & $\% \mathrm{fi}$ & $3,7 \%$ & $7,4 \%$ \\
\hline \multirow{2}{*}{$\begin{array}{l}\text { Tiene internet } \\
\text { en casa }\end{array}$} & No cuentan & $\% \mathrm{fi}$ & $51,9 \%$ & $44,4 \%$ \\
\hline & Cuentan con internet & $\% \mathrm{fi}$ & $48,1 \%$ & $55,6 \%$ \\
\hline \multirow{2}{*}{ Libros de casa } & No tiene libros en casa & $\% \mathrm{fi}$ & $40,7 \%$ & $29,6 \%$ \\
\hline & Si tiene libros en casa & $\% \mathrm{fi}$ & $59,3 \%$ & $70,4 \%$ \\
\hline \multirow{5}{*}{$\begin{array}{l}\text { Grado de } \\
\text { instrucción del } \\
\text { padre*Sección }\end{array}$} & Analfabeto & $\% \mathrm{fi}$ & $0,0 \%$ & $3,7 \%$ \\
\hline & Primaria incompleta & $\% \mathrm{fi}$ & $11,1 \%$ & $0,0 \%$ \\
\hline & Primaria completa & $\% \mathrm{fi}$ & $14,8 \%$ & $11,1 \%$ \\
\hline & Secundaria incompleta & $\% \mathrm{fi}$ & $70,4 \%$ & $74,1 \%$ \\
\hline & Superior incompleto & $\% \mathrm{fi}$ & $3,7 \%$ & $11,1 \%$ \\
\hline
\end{tabular}

Fuente: Elaboración propia

Las comparaciones porcentuales de las variables latentes entre los grupos de estudio se ejecutaron para tener el control de las alteraciones que pudieran afectar el producto de la aplicación del ABP en la comprensión literal. 
a) En los datos de la Tabla 2, sobre la lectura en casa se observa que en el grupos experimental el 22,2\% no leen, el 74,1\% algunas veces y 3,7\% leen todos los días, mientras que en el grupo de control el 18,5\% no leen, el 74,1\% leen algunas veces y 7,4\% leen todos los días. Para comprobar la significancia, se hizo la prueba de Kruskal-Wallis que equivale 0,2 a un valor de significación estadística de 0,595 mayor al nivel de significación de la prueba 0,05 ( $\mathrm{p}$-valor $>\alpha$ ). Lo que significó que la lectura que efectúan en casa, no afectó el producto significativo del ABP en la comprensión literal como se observa en la Tabla 3.

TABLA 3

Nivel de significación de la comprensión literal con la lectura que desarrolla en casa el estudiantado.

\begin{tabular}{|c|c|c|c|c|}
\hline \multicolumn{5}{|l|}{ Rangos } \\
\hline & Test & $\mathrm{N}$ & $\begin{array}{l}\text { Rango } \\
\text { promedio }\end{array}$ & H de Kruskal-Wallis \\
\hline \multirow[t]{3}{*}{ Lee en casa } & Pre test control & 27 & 28,37 & $\begin{array}{l}\text { H de Kruskal-Wallis = } \\
282\end{array}$ \\
\hline & $\begin{array}{l}\text { Pre test } \\
\text { experimental }\end{array}$ & 27 & 26,63 & $\mathrm{gl}=1$ \\
\hline & Total & 54 & & Sig. Asintót $=, 595$ \\
\hline
\end{tabular}

Fuente: Elaboración propia

b) En la tenencia del internet en casa, en el grupo experimental el 51,9\% no cuenta con este servicio y el $48,1 \%$ si cuenta con internet; en el grupo de control el $44,4 \%$ no cuenta con este servicio mientras que el 55,6\% si dispone de ello. Los datos mostrados en ambos grupos no fueron tan dispersos, teniendo las mismas condiciones en la interacción con los textos virtuales. La significancia estadística de Kruskal-Wallis alcanza 0,589 siendo mayor a la prueba 0,05 ( $\mathrm{p}$-valor $>\alpha$ ). Lo que significa que no afectó al tratamiento de la variable en estudio como precisa en la Tabla 4.

TABLA 4

Nivel de significación de la comprensión lectora y el internet que tiene en casa el estudiantado.

Rangos
\begin{tabular}{|lllll}
\hline & Test & $\mathrm{N}$ & Rango promedio & H de Kruskal-Wallis \\
\hline $\begin{array}{l}\text { Tiene } \\
\text { internet en } \\
\text { casa }\end{array}$ & Pre test control & 27 & 28,50 & $\begin{array}{l}\text { H de Kruskal-Wallis } \\
=, 291 \\
\end{array}$ \\
& $\begin{array}{l}\text { Pre test } \\
\text { experimental } \\
\text { Total }\end{array}$ & 27 & 26,50 & $\mathrm{gl}=1$ \\
& 54 & & Sig. Asintótica = ,589 \\
\hline
\end{tabular}

Fuente: Elaboración propia

c) En lo referente a los libros que tiene el estudiantado en casa, se observó que en el grupo experimental el $59,3 \%$ cuenta con este material y el $40,7 \%$ no tiene, frente al grupo de control que el $70,4 \%$ tiene y el $29,6 \%$ no tiene. La significancia estadística de Kruskal-Wallis alcanzó 0,397 siendo mayor a la prueba 0,05 (p-valor $>\alpha$ ) lo cual significa que la tenencia de libros en casa no afecta la aplicación del estudio como se puede observar en la Tabla 5. 
TABLA 5

Nivel de significación de la comprensión lectora y libros que tiene en casa el estudiantado.

Rangos
\begin{tabular}{|lllll}
\hline & & & \\
& Test & Rango & H de Kruskal-Wallis \\
Libros de casa & Pre test control & 27 & 29,00 & H de Kruskal-Wallis $=$ \\
& & & 717 \\
& $\begin{array}{l}\text { Pre test } \\
\text { experimental }\end{array}$ & 27 & 26,00 & gl $=1$ \\
Total & 54 & & Sig. Asintótica $=, 397$ \\
\hline
\end{tabular}

Fuente: Elaboración propia

d) En cuanto al grado de instrucción del padre. En el grupo experimental, el 11,1\% de padres tiene primaria incompleta, $14,8 \%$ primaria completa, $70,4 \%$ de padres tienen secundaria incompleta, $3,7 \%$ de padres tiene superior incompleto. Mientras que, el grupo de control 3,7\% de padres son analfabetos, $11,1 \%$ de padres tiene primaria completa, $74,1 \%$ de padres tienen secundaria incompleta, $11,1 \%$ de padres tiene superior incompleto Según la prueba Kruskal-Wallis, no hay significación en relación con el grado de instrucción del padre, el valor equivale 0,2 a un valor de significación estadística de 0,188 mayor al nivel de significación de la prueba 0,05 ( $\mathrm{p}$-valor $>\alpha)$ como se precisa en la Tabla 6.

TABLA 6

Nivel de significación de la comprensión lectora del estudiantado con el grado de instrucción del padre.

Rangos
\begin{tabular}{|lllll}
\hline & Tes & N & $\begin{array}{l}\text { Rango } \\
\text { promedio }\end{array}$ & H de Kruskal-Wallis \\
\hline Grado de & Pre test control & 27 & 29,72 & H de Kruskal-Wallis = \\
instrucción del & & & & $\begin{array}{l}1,736 \\
\text { gl }=1\end{array}$ \\
padre & Pre test & 27 & 25,28 & Sig. Asintót $=, 188$ \\
& experimental & & & \\
& Total & 54 & &
\end{tabular}

Fuente: Elaboración propia

\section{Discusión}

El objetivo de la investigación es verificar los efectos del ABP en la comprensión literal del estudiantado del $4^{\circ}$ correspondientes IV ciclo de la Educación Básica Regular. En los resultados del pre test se visualizan las dificultades en la comprensión literal, en el grupo de control el 55,56\% se encuentran en el nivel de inicio y $44,44 \%$ en proceso y en el grupo experimental el $59,26 \%$ en inicio y $40,74 \%$ en proceso y es preciso mencionar que ningún estudiante alcanza el logro destacado. Sabiendo que el nivel literal es la base y tiene la facultad de favorecer el dominio de las estructuras semánticas básicas como: la identificación de detalles, secuencias, contenidos principal o secundario en la información explicita del texto que sustentan los otros niveles y con ello las habilidades de orden superior.

Los resultados obtenidos en el post test del grupo experimental reflejan que la aplicación del Aprendizaje Basado en Problemas de John Barell, tiene efecto positivo en la comprensión literal, al promover el interés por la lectura para resolver los retos planteados, donde, el $74 \%$ de estudiantes se ubica en la escala más alta, como el logro destacado y, el 25,93\%, el logro esperado, resultados se apoyan en otras investigaciones (Peña 2019 y Durango 2017), al sustentar que el alumnado disfruta más la lectura si conocen su propósito, 
observan el panorama de su contenido, comentan sus entendimientos previos y, finalmente, discuten sobre la intencion del autor que está plasmado entre los elementos oracionales de manera explícita. Nunura (2018), sustenta la influencia significativa en la comprension literal en su trabajo de investigación Estrategias de la jerarquización de la informacion donde el grupo experimental obtiene en el pre test $52 \%$ de deficiencia en la lectura literal y, al pasar al post test, alcanza el 52\% nivel bueno. Fonseca et al. (2019), en su investigacion cuasi experimental, lee comprensivamente, basado en la enseñanza explícita de habilidades de alto nivel vinculados al procesamiento de de texto, en los hallazgos de su intervencion describe mejoras significativas en la comprension lectora de niños dentro de la escuela.

Es imprescindible que todo docente se involucre en la búsqueda y la aplicación de nuevas estrategias o método de enseñanza que faciliten superar las dificultades de la comprensión literal. Como se contempla en la información del trabajo desarrollado en el grupo de control el 85,18\% aún permanecen en los dos niveles de logro más bajos (inicio $=62,18 \%$ ) y (proceso $=22,22 \%$ ), en confrontación al grupo experimental que recibió el tratamiento no registrando ningún dato en estos niveles de logro por la efectividad favorable del estudio. Sabiendo que hay mucha dificultad en la comprensión literal, como en los estudios citados. De Mier, et al. (2015), mencionan en su estudio que estudiantes del $4^{\circ}$ grado, a medida que avanzan en su escolaridad, tienen mayor experiencia con los textos expositivos y, a pesar de ello, tienen dificultad para recuperar la información literal. Vidal y Manríquez (2016), revelan en su estudio el bajo nivel de comprensión de textos escritos, motivo por el cual los formadores deben encaminar esta actividad con asistencia de estrategias. Rojas y Cruzata (2016), en la investigación organizada en el nivel primario menciona que docentes trabajan de manera empírica y con modelos tradicionales incidiendo en la baja comprensión literal: como el 16,7\% se encuentran en el nivel inicial evidenciando dificultades y mayor acompañamiento por parte del profesorado, el $25,0 \%$ en proceso, el $50 \%$ en logro previsto y solo el 8,3\% en logro destacado demostrando el manejo solvente de las tareas propuestas. Frente a lo descrito se ratifica que hay mucha dificultad en la comprensión literal en estudiantes y es necesario emplear otros métodos o estrategias que promuevan la comprensión lectora y el manejo solvente de la gran cantidad de información que se tiene en un mundo globalizado.

$\mathrm{El} \mathrm{ABP}$ es efectivo en la comprensión lectora como lo demuestran los resultados, porque se sustenta en un enfoque investigativo, exigiendo a cada estudiante que se involucre en la lectura, para buscar repuestas de las situaciones problemáticas contextualizadas que se le plantea generando dudas e incertidumbre y convirtiéndolas en significativas y retadoras. Rigo y Donolo (2017), en su investigación llega a la conclusión de que el aprendizaje es positivo cuando se vincula a la vida cotidiana y se incrementa con la investigación al permitir que estudiantes aprenden a buscar información, con mayor autonomía, y favorece la toma de decisión. Vidal y Manriquez (2016), en su estudio desarrollado manifiesta que hay bajo nivel de la comprensión literal en todos los niveles educativos, motivo por el cual cada docente debe guiar esta práctica a través de estrategias de enseñanza que apoyen a la revisión de textos. Teniendo en cuenta los estudios mencionados es pertinente que el estudiantado se involucre con la lectura de diversos textos tal cual lo propone el $\mathrm{ABP}$ en los retos que plantea donde cada docente es orientador/a y el alumnado tiene el protagonismo.

\section{ConCLUSIón}

Teniendo en cuenta los sustentos teóricos del trabajo desarrollado sobre la comprensión literal, la cual consiste en extraer la informacion que se encuentra de manera explícita en cualquier parte del texto. Se observa que el estudiantado del $4^{\circ}$ grado tiene dificultaded, como lo demuestran los resultados del pre test de ambos grupos y el pos test del grupo de control de la investigación efectuada y los hallazgos encontrados en otros estudios. Por tanto es recomendable la aplicación de la metodologia del ABP de John Barell, la cual permite impulsar los logros significativos en la comprension literal en estudiantes del $4^{\circ}$ grado correspondientes al IV ciclo de la Educación Básica Regular, donde el aprendiz indaga el reto proyectado de 
manera desafiante y significativo que le permite la interacción con el texto según su propósito lector y en esta interacción con el texto comienza a identificacar detalles, discriminar secuencias, reconocer contenidos principales y secundarios del texto, teniendo la posibilidad de explorar mucho más allá de lo explícito por la demanda cognitiva alta que requieren los retos propuestos en cada paso secuencial del ABP hasta llegar a debates, aplicación en otras circuntancias o compromisos en la solucion de casos propuestos y los nuevos retos que surgen por el interés del escolar.

\section{REFERENCIAS}

Barell, J. (2007). El aprendizaje basado en problemas: Un enfoque Investigativo. Buenos Aires: Manatial.

Campbell, D., y Stanley, J. (1995). Diseños experimentales y cuasi experimentales en la investigación social. Buenos Aires: Amorrortu editores.

Campos, D., Contreras, P., Riffo, B., y Véliz, M. (2014). Complejidad textual, lecturabilidad y rendimiento lector en una prueba de comprensión en escolares adolescentes.JAVERIANA, 13(3). doi: https://doi.org/10.11144/Jav eriana.UPSY13-3.ctlr

Carranza, M. D., y Caldera, J. (2018). Percepción de los estudiantes sobre el aprendizaje significativo y estrategias de enseñanza en el Blended Learning. REICE. Revista Iberoamericana sobre Calidad, Eficacia y Cambio en Educación, 16(1), 73-88. doi: https://doi.org/10.15366/reice2018.16.1.005

De Mier, M., Amado, B., y Benítez, M. (2015). Dificultades en la Comprensión de Textos Expositivos en Niños de los Primeros Grados de la Escuela Primaria. PSYKHE, 24(2), 1-13. doi: https://doi.org/10.7764/psykhe.24.2.708

Durango, Z. (2017). Niveles de comprensión lectora en los estudiantes de la Corporación Universitaria Rafael Núñez (Cartagena de Indias). Revista Virtual Universidad Católica del Norte, 51, 156-174. doi: http://dx.doi.org/10. $18271 /$ ria.2015.137

Flavell, J. (1979). Metacognition and cognitive monitoring: A new area of cognitive-developmental inquiry. American Psychologist, 34(10), 906-911. doi: http://dx.doi.org/10.1037/0003-066X.34.10.906

Fonseca, L., Migliardo, G., Simian, M., Olmos, R., y León, J. A. (2019). Estrategias para Mejorar la Comprensión Lectora: Impacto de un Programa de Intervención en Español. Psicología Educativa, 25(2), 91-99. doi: https:/ /doi.org/10.5093/psed2019a1

López, S., Veit, E., y Solano, I. (2014). La formulación de preguntas en el aula de clase: una evidencia de aprendizaje significativo crítico. Ciência y Educação, Bauru, 20(1), 117-132. doi: https://doi.org/10.1590/1516-73132014 0010007

Malla, N. (2016). Pautas de selección de materiales curriculares en la enseñanza del inglés como lengua extranjera. Revista Complutense de Educación, 27(2), 707-724. doi: https://doi.org/10.5209/rev_RCED.2016.v27.n2.478 53.

Marra, R., Jonassen, D. H., Palmer, B. y Luft, S. (2014). Why problem- based learning works: Theoretical foundations. Journal on Excellence in College Teaching, 25(3-4), 221-238. Recuperado de https://www.albany.edu/cee/asset s/Why_Problembased_learning_works.pdf

Ministerio de Educación del Perú. (2018). Evaluación de logros de aprendizaje. Oficina de Medición de la calidad de los aprendizajes (UMC) Recuperado de https://bit.ly/2wgu6Ji

Morales, P. (2018). Aprendizaje basado en problemas (ABP) y habilidades de pensamiento crítico ¿una relación vinculante? Revista Electrónica Interuniversitaria de Formación del Profesorado (REIFOP), 21(2), 91-108. doi: h ttps://doi.org/10.6018/reifop.21.2.323371

Novoa, P., Cancino, R., Flores, W., y Nieto, J. (2018). The Harmonic Mind Map in the Comprehension of Narrative Texts in University Students. Propositos y Representaciones, Revista de Psicologia Educativa, 6(2), 541-606. doi: https://doi.org/10.20511/pyr2018.v6n2.243 
Nunura, T. (2018). Estrategias de jerarquización de la información en los niveles de comprensión lectora en estudiantes de Ingeniería Industrial. Trujillo - 2017. SCIÉNDO, 21(4), 507-513. doi: https://dx.doi.org/10.17268/sciend o.2018.057

Peña, S. (2019). El desafio de la comprensión lectora en la educación primaria. Revista Panorama, 13(24), 43-56. doi: htpps://doi.org/10.15765/pnrm.v13i24.1205

Pinzas, J. (2003). Se aprende a leer, leyendo. Ejercicios de comprension de lectura para los docentes y sus estudiantes. Lima, Perú: Tarea asociación de publicaciones educativas.

Pinzas, J. (2017). Leer pensando Introducción a la visión contemporánea de la lectura. Lima, Perú: Fondo Editorial Pontificia Universidad Católica.

Ramos, L., y Casas, L. (2018). Demanda Cognitiva de Estándares Educativos y Libros de Texto para la Enseñanza del Álgebra en Honduras. Bolema: Boletim de Educação Matemática, 32(62), 1134-1151. doi: http://dx.doi.org/1 $0.1590 / 1980-4415 \mathrm{v} 32 \mathrm{n} 62 \mathrm{a} 19$

Rigo, D., y Donolo, D. (2017). El valor de utilidad de los contenidos escolares. Percepciones de los estudiantes de nivel primario. Revista psicodebate:psicologia, cultura y sociedad, 17(1), 51-69. Recuperado de https://bit.ly/2OrMzeU

Rodríguez, C., Fernández, J. (2017). Evaluación del Aprendizaje Basado en Problemas en Estudiantes Universitarios de Construcciones Agrarias. Formación universitaria, 10(1), 61-70. doi: https://dx.doi.org/10.4067/S0718-50 062017000100007

Rojas, C., y Cruzata, M. (2016). La comprensión lectora en estudiantes de educación primaria en Perú. Revista de Educación, (9), 337-356. Recuperado de https://fh.mdp.edu.ar/revistas/index.php/r_educ/article/view/1916/ 1854

Ruvalcaba, N., Gallegos, J., Orozco, M., y Bravo, H. (2019). Validez predictiva de las competencias socioemocionales sobre la resiliencia en adolescentes mexicanos. Diversitas, 15(1), 87-99. Recuperado de https://revistas.usantot omas.edu.co/index.php/diversitas/article/view/3897

Sánchez, M. (2018). La evaluación del aprendizaje de los estudiantes: ¿Es realmente tan complicada?. Revista Digital Universitaria, 19(6), doi: https://doi.org/10.22201/codeic.16076079e.2018.v19n6.a1

Sastoque, D., Ávila, J., y Olivares, S. (2016). Aprendizaje Basado en Problemas para la construcción de la competencia del Pensamiento Crítico. Revista Latinoamericana de Educación: Voces y Silencio, 7(1), 148-172. doi: http://dx .doi.org/10.18175/VyS7.1.2016.08

Souza, C., Pfeiffer, E., y Mendes de Oliveira, J. (2015). Transferencia de aprendizaje y complejidad de tareas: "La carreta delante de los bueyes". Revista Interamericana de Psicologia/Interamerican Journal of Psychology (IJP). 49(3), 294-301. Recuperado en http://www.redalyc.org/pdf/284/28446020002.pdf

Tamayo, Ó., Cadavid, V., y Montoya, D. (2019). Análisis metacognitivo en estudiantes de básica, durante la resolución de dos situaciones experimentales en la clase de Ciencias Naturales. Revista Colombiana de Educación, 1(76), 117-141. doi: https://doi.org/10.17227/rce.num76-4188

Toro, M. d., y Buitrago, M. (2018). Organizadores gráficos para afianzar competencias lectoras en estudiantes universitarios de primer semestre. Concept Mapping: Renewing Learning and Thinking, Conferencia llevada a cabo en $8^{\circ}$ International Conference on Concept Mapping, Colombia. Recuperado de cmc.ihmc.us/ cmc2018papers/cmc2018-p97.pdf

Vidal, D., y Manríquez, L. (2016). El docente como mediador de la comprensión lectora en universitarios. Revista de la educación superior, 45(177), 95-118. doi: https://doi.org/10.1016/j.resu.2016.01.009

Zeballos, S., y Nyquist, D. (2019). Dificultades en la comprensión lectora y su vinculación con la Teoría de la Doble Ruta. Revista de Difusión cultural y cientifica de la Universidad La Salle en Bolivia, 14(14), 15-33. Recuperado de https://bit.ly/2MkIypT

\section{BY-NC-ND}

